\title{
De la Visualización a la Sensorización de Información
}

\section{From the Visual Information to the Sensing Information}

\author{
Diego Pimentel \\ Instituto Universitario Nacional del Arte, Argentina \\ dpimentel@buenosaires.gob.ar \\ Mariano Cataldi \\ Instituto Universitario Nacional del Arte, Argentina \\ m.cataldi@iuna.edu.ar
}

Gonzalo Muñiz

Instituto Universitario Nacional del Arte, Argentina

contacto.gonzalomuniz@gmail.com

\begin{abstract}
Information Visualization is a discipline implemented on interfaces which use one sense to understand the ideas developed: sight. However, there are physical interfaces that allow users to interpret the data, either through sound or touch. The objective of this paper is to discussed the preliminary results of the research project that frames this paper, as a work in progress.
\end{abstract}

Keywords: Information; Visualization; Interfaces; Design; Infography.

\section{Introducción}

La visualización de información consiste en "la utilización de interfaces interactivas cuya finalidad principal es representar con mínima entropía visual una serie de datos a un usuario final" y se caracteriza por "(1) ser interrelacional, (2) transformar datos crudos en información relevante, (3) buscar la mínima pérdida de información en dicha transformación, y (4) dirigirse a usuarios que interactúan, transforman e interpretan esta información" (Córdoba Cely y Alatriste Martínez: 2012).

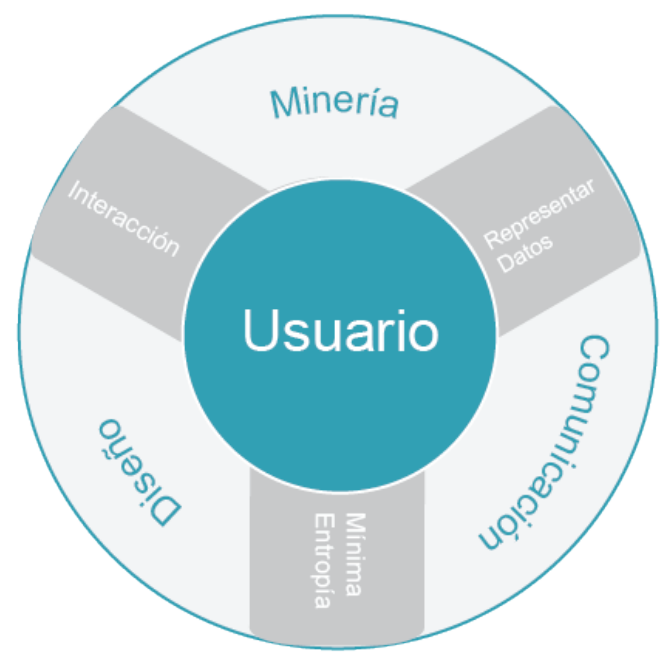

Figura 1: Características limitantes de la $\mathrm{VI}$ y relaciones con otras disciplinas. Referencia:

http://www.nosolousabilidad.com/articulos/taxonomia_visualizacion.htm, Córdoba Cely, C. / Alatriste Martínez, Y. (2012): Hacia una taxonomía de investigación entre Visualización de Información y Diseño. Publicado en la revista electrónica no solo usabilidad: revista multidisciplinar sobre personas, diseño y tecnología (ISSN 1886-8592), consultado el 10/11/12.

Ben Fry, en sus tesis que da origen al Processing como lenguaje de programación, Computational Information Design (2004), desarrolla un proceso de entendimiento de datos utiliza siete elementos en un proceso en el cual se integran la computación científica, la matemática, estadística y minería de datos, el diseño gráfico, la visualización de la información y la $\mathrm{HCl}$ (Human Computer Interaction). Estas siete palabras clave que coexisten en el proceso son: adquirir, analizar, filtrar, minar, representar, refinar e interactuar.

Estas exploraciones iniciales finalizaron con la tesis de referencia Computational Information Design (2004)- a partir de la cual se establecieron parámetros de análisis para la construcción de obras que toman a la información como punto de partida, y que luego transforman su presentación de acuerdo al sentido estético que se pretende otorgar.

Un ejemplo de estos resultados es similar Diversity (Fig. 3), un proyecto desarrollado en Processing que pretende establecer las relaciones entre las cinco religiones más importantes de nuestro contexto contemporáneo, a través de tomar información de los textos sagrados de cada una de ellas y luego, mostrando las palabras que más se repiten en cada uno de estos textos, utilizando una estructura cromática y morfológica, que pone de manifiesto la complejidad de relaciones entre las mismas. 


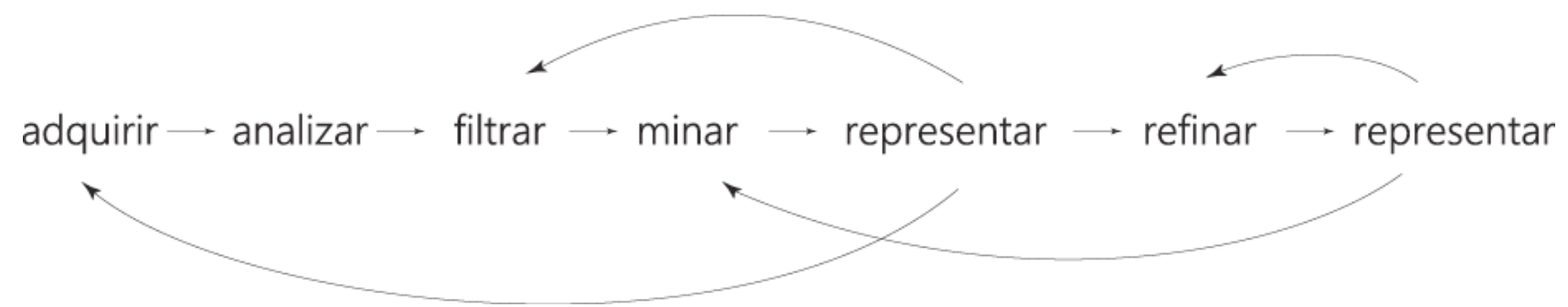

Figura 2: Esquema de proceso de entendimiento de datos. Fry, B. (2004): Computational Information Design, Cambridge: MIT Press, p. 30. Traducción realizada por los miembros del proyecto.

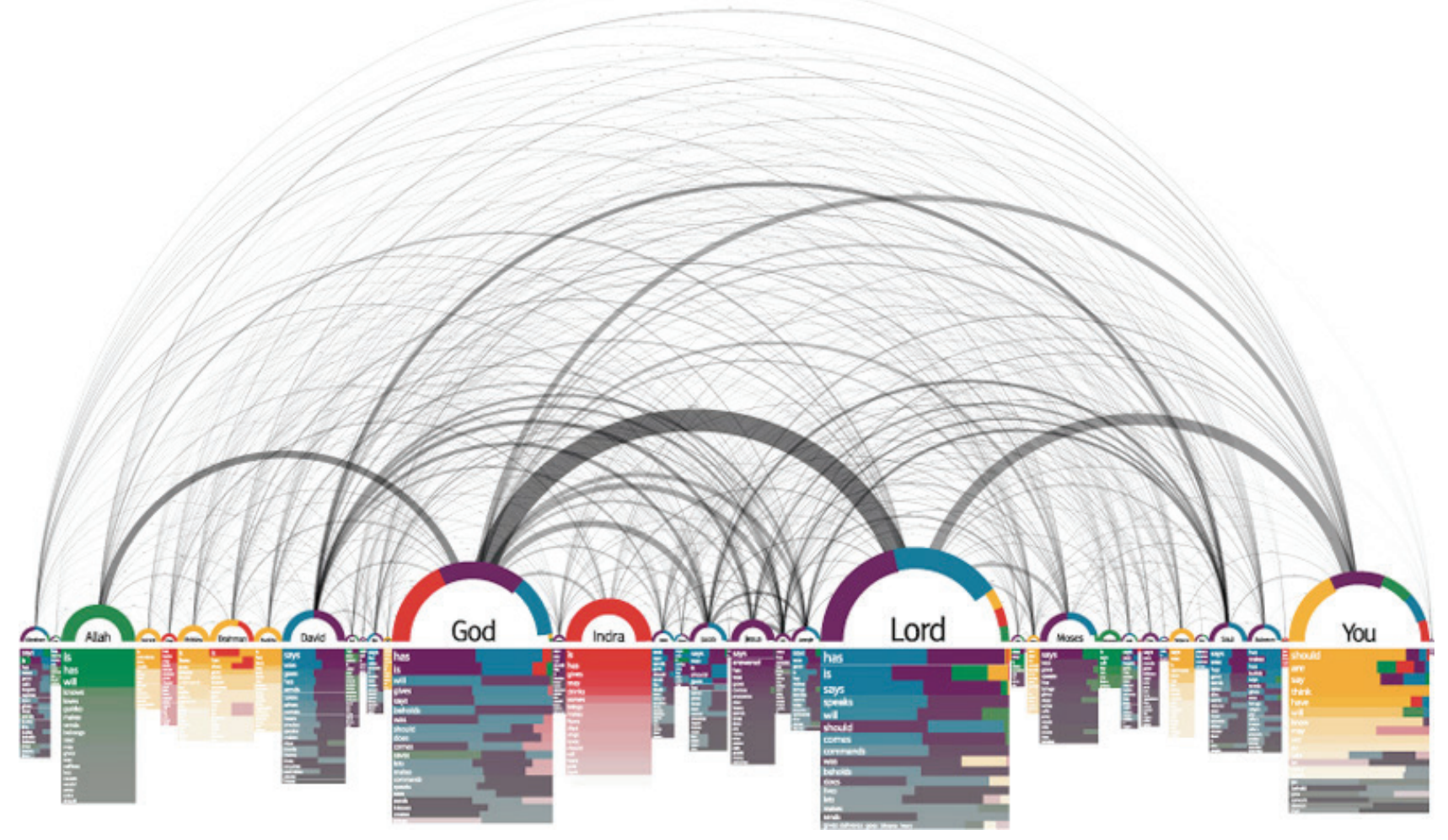

Figura 3: Similar Diversity (2007). Steinweber, P. y Koller, A.

\section{Antecedentes Históricos}

Sin embargo, los antecedentes históricos se pueden encontrar en ejemplos de visualización en la era "pre-computadoras":

- La inclinación de las órbitas planetarias - siglo X

- Índices de importación-exportación de mercancías (Playfair) - siglo XVIII

- Cartografías de la variación magnética terrestre (Halley) - siglo XVIII

- Campaña de Napoleón en Rusia (Minard) - siglo XIX

- $\quad$ Epidemia de Cólera en Londres (Snow) - siglo XIX

\section{El Diálogo Proyectual}

“¿Por qué deberíamos estar interesados en la visualización? Debido a que el sistema de visualización humano es un buscador de patrón de enorme poder y sutileza. El ojo y la corteza visual del cerebro forman un procesador paralelo masivo que proporciona el canal de mayor ancho de banda en los centros humanos cognitivos. A mayores niveles de procesamiento, la percepción y la cognición están estrechamente relacionadas entre sí, razón por la cual las palabras "comprensión" y "ver" son sinónimos." (Ware, 2000).

La visualización ayuda al conocimiento, amplía la capacidad humana para entender. Esta idea expresada por teóricos contemporáneos que reflexionan en el cruce de las artes, la 
cultura y la tecnología, tiene sus antecedentes no sólo en las disciplinas mencionadas en la definición de la visualización de información (artes visuales, el diseño gráfico, la tipografía, la minería de datos, la computación científica, la semiótica y el periodismo) sino también en otros referentes de la cultura, entre los cuales destacamos el concepto de visibilidad de Italo Calvino.

Calvino (1985) define la visibilidad como una de las constantes para la literatura en su escrito "seis propuestas para el próximo milenio". Para Calvino la visibilidad no implica la propiedad de ver "a través de", sino la capacidad de "imaginar", de "ver" en la mente una idea. Para desarrollar su concepto, recorre y relaciona textos de Dante Alighieri, Samuel Taylor Coleridge y Jorge Luis Borges, demostrando que en todos los casos, durante el desarrollo de las obras estos autores recurrieron al menos en un momento, a instancias por fuera del proceso racional o sistemático. Por ejemplo, cita un fragmento de la Divina Comedia, según Calvino, Dante se arroga el poder de dialogar con Dios:

O imaginative che ne nube

talvolta sí du fuor, ch.om non s.accorge

perché dintorno suonin mille tube,

chi move te, si $\cdot$ l senso non ti porge?

Moveti lume che nel ciel s.informa

per sé o per voler giú le sorge.

(Oh fantasia que, de de cuando en cuando, / arrebatas al hombre de tal suerte/que no oyera mil tubas resonando,/ ¿quién, si no es el sentido ha de moverte?/ Muévete aquella luz que el cielo sella., /por si o por el querer de quien la vierte.)

En esta línea argumentativa, Dante estaba convencido que dialogaba con Dios -el querer que quién la vierte- y que su misión terrenal consistía en escribir la conformación conceptual, morfológica y simbólica del cielo y el infierno.

En relación con Calvino, y relacionada a la idea de la visibilidad, es importante mencionar a los teóricos del pensamiento proyectual, dentro de los cuales se destaca Bruno Munari, quién analiza el proceso que un diseñador entabla en el transcurso de la creación, desde la idea, el relevamiento de necesidades, los materiales utilizados, la experimentación, verificación posterior y finalmente la construcción.

En el citado "proceso proyectual" definido por Munari (1990), se presenta al mismo como una semirrecta, con inicio, pero sin fin. Este proceso inicia cuando se plantea el problema: qué se debe diseñar y finaliza cuando se arriba a la solución: el diseño concluído. Al proceso proyectual, basado en el par problemasolución, entre uno y otro extremo se encuentra la instancia creativa (Fig. 4). Para Munari esa instancia creativa se encuentra a mitad de camino entre el problema y la solución, en palabras de Calvino, en un momento del proceso el diseñador "visualiza" la solución de un problema. Sin embargo, a partir de esa visualización del problema, el diseñador debe enfrentar el proceso de traducción entre su interioridad y el resultado del proceso: el diseño terminado.

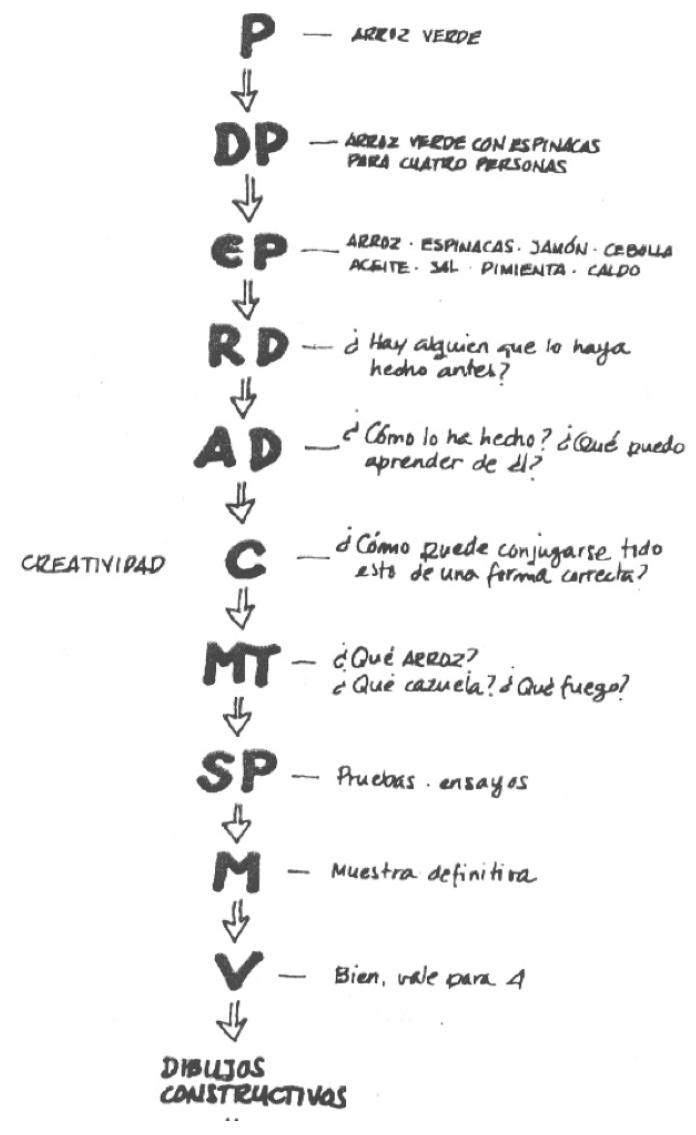

Figura 4: Diagrama del proceso proyectual. Munari, B. (1990): Cómo nacen los objetos. Barcelona: Gustavo Gili.

A su vez, el proceso representado por Munari, encuentra un paralelo, más complejo, en la tesis de Ben Fry (2004) Computational Information Design, quien utiliza siete elementos en un proceso en el cual se integran la computación científica, la matemática, estadística y minería de datos, el diseño gráfico, la visualización de la información y la $\mathrm{HCl}$ (Human Computer Interaction). Estas siete palabras clave que coexisten en el proceso son: adquirir, analizar, filtrar, minar, representar, refinar e interactuar (Fig.7).

La relación entre Munari y Fry es notable. Ambos teóricos plantean sus ideas como una semirrecta que vuelve, necesariamente, sobre sus pasos en más de una oportunidad, redefiniendo ideas que anteriormente habían sido establecidas.

\section{De la Vista a un Sensorio más Amplio}

Si bien la visualización de información se centra en el conjunto de relaciones que permiten al usuario transformar y sintetizar una 
determinada cantidad de información a partir de una base de datos, la Visualización de Datos se orienta a la representación y análisis visual de esos datos tratados en gráficos estadísticos y cartografía temática (Friendly; 2009). Sin embargo, los límites entre visualización de información, visualización de datos y visualización científica son borrosos (Sousa Santos; 2010).

Como referencias finales, es importante mencionar un referente histórico, el mapa de metro de Londres (Beck, 1933), Stars (Google Chrome Experiments, 2012) y la interfase física de realidad mixta The Rhythm of Cities (Canet, M. y Guljajeva, V., 2012), tres ejemplos de visualización de información que cruzan ciencia, diseño, arte y tecnología.

The Rhythm of City (2011) es una obra de realidad mixta en tiempo real realizada por Mar Canet y Varvara Guljajeva, utilizando datos de geolocalización de Twitter, Flickr y Youtube con propósitos artísticos. Una placa arduino conecta la información de cada ciudad en relación al uso de redes sociales, modificando el ritmo de cada metrónomo. Este ejemplo de interfase física no se centra en información mostrada visualmente, sino que -utilizando datos- la visualización de la información está interpretada por distintos sentidos: el ojo percibe el movimiento diferente de cada metrónomo, pero a su vez, el ritmo de los mismos está semantizado por el sonido generado. A su vez, el espacio de la instalación genera una

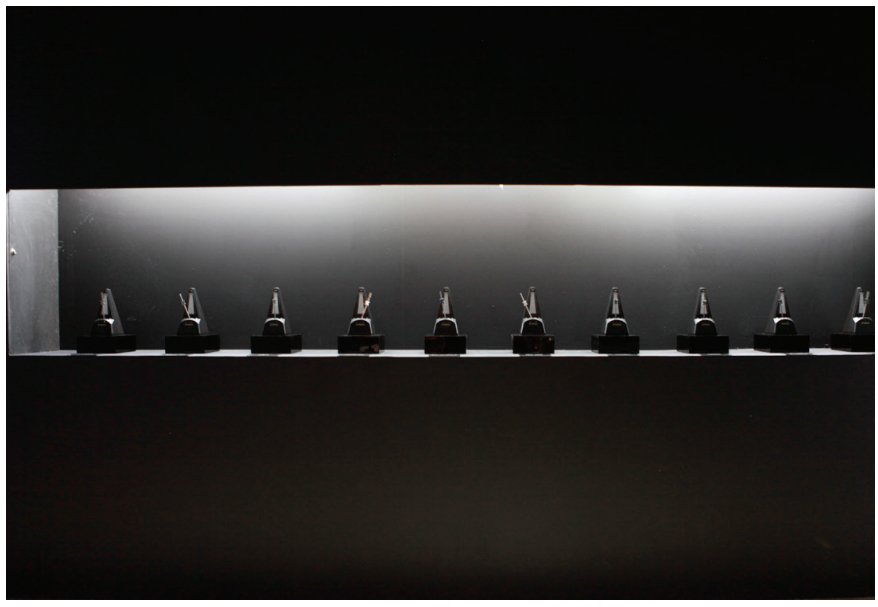

Figura 5. The Rhythm of City. Canet, M. y Guljajeva, V., obra de realidades mixtas. 2012, Centro Cultural San Martín, Buenos Aires.

\section{Conclusiones preliminares}

Los primeros avances del proyecto de investigación en el cual se encuentra enmarcado este paper se orientan a la construcción de un lenguaje en común por parte de los miembros del equipo, de origen disciplinar diverso, con la recopilación de material de lectura y referentes que hemos puesto en común. El propósito final de este proyecto de investigación es consolidar los mecanismos conceptuales, técnicos y metodológicos para la visualización de información con este tipo de instalaciones sensoriales, trascendiendo la mera utilización de lo visual como única forma representacional de información.

Nuestros pasos subsiguientes se enmarcan en las siguientes líneas de trabajo:

\section{Relevamiento de herramientas de software y técnicas existentes en relación a:}

- Sistemas de simulación física, simulación de sistemas complejos, interfaces digitales en web, interfases físicas o cualquier otro tipo de producción lineal o interactiva, en torno a la visualización de información.

- Sistemas de Visualización de Datos e información en tiempo real.

- Diseño participativo y colectivo. Ejemplos de UX (User Experience) en casos de visualización de datos complejos.

- Cualquier otro algoritmo o sistema que permita la creación de Visualizaciones de Datos mediante la adopción de estrategias pertenecientes al género.

- Minería de datos, sus metodologías e impactos en la visualización de la información.

\section{Elaboración de un marco conceptual que permita:}

- Definir la clasificación de los diferentes tipos de experiencias artísticas interactivas relacionadas a Visualización de datos, adoptando diferentes criterios (algoritmos, formatos, etc.), para crear un taxonomía que permita identificar rasgos constitutivos, a nivel temático, retórico y enunciativo, en obras que hagan uso de la Visualización de la información.

- Abordar la especificidad de la Visualización de Datos vinculando a las Artes Electrónicas/Digitales y los Medios digitales, así como con otros campos de conocimiento.

- Establecer criterios de diseño para el abordaje de una Obra que incluya experiencias sobre Visualización de información.

- Interfases de sensorización: Datos y percepción, Metáforas y desarrollo de sistemas de visualización, la sensorización de la información como amplificadora de conocimiento. 


\section{Referencias}

Bertin, J. (1983). Semiology of Graphics: Diagrams, Networks, Maps. Madison, Wis.: University of Wisconsin Press.

Bonsiepe, G. (1998). Del objeto a la interfase. Mutaciones del diseño. Editorial Infinito, Buenos Aires.

Calvino, I. (1989). Seis popuestas para el próximo milenio. Madrid: Siruela, Madrid.

Card, S., Mackinlay, K., Jock, D. \& Shneiderman, B. (1999). Readings in Information Visualization: Using Vision to Think. San Francisco: Morgan Kauffman.

Grinstein, G. \& Ward, M. (2002). Introduction to Data Visualization. En Information Visualization in Data Mining \& Knowledge Discovery.

Hand, D., Manila, H. \& Smyth, P. (2001). Principles of Data Mining (Adaptive Computation and Machine Learning). Cambridge, mit Press.

Lima, M. (2011). Visual Complexity: Mapping Patterns of Information

Klanten, R., Bourquin, N., Ehmann, S., van Heerden, F., Tissot T. (2008). Data Flow: Visualising Information in Graphic Design. Gestalten.

Finke T., Manger S. \& Fichtel S. (2012). Informotion: Animated Infographics. Gestalten.

Friendly, M. \& Denis, D. (2001). Milestones in the history of thematic cartography, statistical graphics, and data visualization. En: York University, Milestones Proyect. [Consultado: November 11, 2012]. Disponible en: <http://www.datavis.ca/milestones/>.

Friendly, M. (2009). Milestones in the history of thematic cartography, statistical graphics, and data visualization. National Sciences and Engineering Research Council. Canada: Grant OGP0138748.
Fry, B. (2004). Computational information design. PhD. Thesis. Massachusetts Institute of Technology, Program in Media Arts \& Sciences.

Munari, B. (1990). Cómo nacen los objetos. Barcelona: Gustavo Gili

Rheingold, H. (2012). Mind amplifier. Can Our Digital Tools Make Us Smarter?. Ted Books.

Shiffman, D. (2012). The nature of code. Versión gratuita (con licencia de Creative Commons): http://natureofcode.com/book/

Sousa Santos, B. (2010). Introduction to Data and Information Visualization, consultado el $8 / 11 / 12$ en http://www.ieeta.pt/ bss/MAPI/Introduction-to-Vis-5-10.pdf

Steele, J. (2010). Beautiful Visualization: Looking at Data through the Eyes of Experts (Theory in Practice)

Tufte, E. (1990). Envisioning Information. Cheshire, Conn.: Graphics Press.

Tufte, E. (1992).The Visual Display of Quantitative Information. Cheshire, Conn.: Graphics Press.

Tukey, J.W. (1977). Exploratory Data Analysis. Reading, ma: AddisonWesley.

Tukey, J.W. (2001). The Future of Data Analysis, Ann. Math. Statist., 33, 167. Venter et al. Science 291(5507):1304-1351.

Ware, C (2000). Information Visualization: Perception for Design. NY: MK Pres 